Bolm Inst. oceanog., S Paulo, 34 (ünico):79-85, 1986

\title{
ESTUDO SOBRE A ICTIOFAUNA ACOMPANHANTE DA PESCA DO CAMARÃO SETE-BARBAS (Xyphopenaeus kroyeri) NAS PROXIMIDADES DA BAIA DE SANTOS - SP, I - ASPECTOS QUANTITATIVOS
}

Alfredo Martins PAIVA-FILHO \& João Marcos Miragaia SCHMIEGELOW*

Instituto Oceanogräfico da Universidade de São Paulo (Caixa Postal 9075, 01051 São Paulo, SP)

\section{Synopsis}

During the sea-bob shrimp fishery, a large quantity of miscellaneous fishes are caught, of which the most part was discarded in the sea. In this paper, quantitative aspects of this bycatch products are analized, e.g. diversity of ichthyofauna, catch per unit effort and proportions of the sea-bob shrimp versus acompanying fish fauna. Sampling has been made from Sspt. 1981 to Sept. 1983 at Santos bay, one of the most important fishing area from Brazil. A total of 5583 fishes were collected during 28 fishery operations with otter-trowt.

Descriptors: Bycatch, Shrimp fisheries, Xyphopenaeus kroyeri, Marine fishes, Catch composition, Catch/Effort, Santos Bay: SP, Brazil.

Descritores: Fauna acompanhante, Pesca de camarão, Xyphopenaeus kroyeri, Peixes marinhos, Composição da captura, Produção/Esforço, Baía de Santos: SP.

\section{Introdução}

$\mathrm{Na}$ pesca do camarão sete-barbas (Xyphopenaeus kroyeri), são capturadas grandes quantidades de peixes e estes, na maioria das vezes, são devolvidos mortos ao mar por não haver mercado para sua comercialização.

A nível mundial há um grande interesse em se encontrar mecanismos tecnológicos e econômicos adequados para o aproveitamento da fauna de peixes capturada pelos barcos camaroneiros (CarranzaFrazer \& Grande, 1982), bem como em se tentar conhecer as possíveis alterações na cadeia alimentar marinha, devido a destruição de peixes jovens.

Do ponto de vista ecológico, o conhecimento desta fauna è de importância fundamental, já que esta pesca de arrasto, de característica nitidamente predatória, pode ser realizada em äreas muito procuradas por indivíduos jovens de diversas espécies de peixes, como o que

(*) Pós-graduando da ārea de oceanografia biológica.

Publ. n. 654 do Inst. oceanogr. da Usp. acontece na baía de Santos. O desenvolvimento de técnicas de pesca, pode diminuir a níveis significativos a captura de peixes nestas operações (Sternin \& A11sopp, 1983).

A pesca do camarão sete-barbas é realizada em grande escala no litoral brasileiro, apresentando uma significativa importância econômica. Sua pesca no 1itoral de São Paulo, se dá principalmente numa área compreendida dentro de 20 milhas da boca da baía de Santos (Neiva, 1969). De 1981 a 1983, os barcos pesqueiros de Santos foram os responsāveis, em média, por $67,3 \%$ do desembarque de camarão sete-barbas em todo o litoral de São Paulo, sendo sobrepujado apenas pela sardinha (Sardinella brasiliensis) (Instituto de Pesca, 1981-1983). Isto equivale a uma captura média anual, em torno de 5000 toneladas de camarão sete-barbas.

0 produto da pesca é variado, sendo constituído, além do camarão sete-barbas e de outras espécies de camarão, por peixes como a pescadinha, corvina, goete e cação (Neiva, 1969). Cálculos globais atuais da pesca mostram a proporção em 
Bolm Inst. oceanogr., S Paulo, 34 (ünico), 1986

peso camarão/peixe de 1:5 em águas temperadas e 1:10 em águas tropicais

(Slavin, 1983).

Embora existam alguns estudos sobre o camarão sete-barbas capturado na região de Santos (Vieira, 1947; Neiva, 1963, 1969; Santos et al., 1969, 1971a,b;

Iwai, 1973), a fauna acompanhante de sua pesca ainda não foi analisada e detalhamente estudada, o que, em parte, é feito neste trabalho.

\section{Material e métodos}

0 material analisado, num total de 5.583 exemplares, foi obtido com o auxîlio do B/Pesq. "Veliger II" do Instituto Oceanogräfico da Universidade de São Paulo.

Foram realizadas 56 operações de pesca no período de 10-09-1981 a

21-09-1983. Estas operações, em número de duas por viagem, ocorreram nas proximidades da baía de Santos, sempre nos locais onde a frota comercial da pesca do camarão sete-barbas estava atuando, utilizando-se para tal, uma rede de arrasto tipo "otter-traw1" com malhagem de $20 \mathrm{~mm}$ entre-nós nas laterais e no corpo e $15 \mathrm{~mm}$ no saco da rede.

De todos os dados coletados durante cada operação de pesca e em laboratório, utilizamos neste primeiro trabalho da série, aqueles referentes a número de espécies, número e peso dos indivíduos mais abundantes, peso total da captura (incluindo peixes, camarões e outros organismos), peso total de peixes e de camarões e duração do arrasto.

A Tabela 1 mostra os dados referentes a cada operação de pesca e parte dos dados amostrais.

Calculou-se a produção por unidade de esforço (PUE) utilizando-se o peso total da captura obtida, dividido pelo tempo de cada arrasto (Tab. 1; Fig. 1).

Com base nos dados obtidos em cada amostra, foi calculado o indice de diversidade de Shannon-Wiener utilizando-se o logarítmo natural, sendo a unidade denominada "natural bel" segundo Good (1950). Sua variação estā representada na Figura 2.

A partir dos dados de peso total dos peixes e dos camarões da amostra, constantes da Tabela 1, foi calculada a porcentagem do peso dos peixes (Fig. 3).

A Tabela 2 mostra as espécies mais freqüentes em número com respectivos pesos, em valores absolutos e em porcenta- gem do total capturado.

A Tabela 3 mostra o número máximo, mínimo e médio de espécies de peixes por arrasto, além da relação camarão/peixe em seus valores máximo, mínimo e médio.

Não houve interesse, no momento, em se avaliar a significância amostral entre os diferentes arrastos. 0 objetivo do presente trabalho se concentrou tão somente na descrição da fauna acompanhante da pesca do camarão sete-barbas. Por essa razão, testes amostrais do tipo "Wilcoxon matched signed rank test" não são ora considerados; sua aplicação, no entanto, poderá ser levada em conta em próximas contribuições sobre o assunto.

\section{Resultados e Discussão}

Em 56 arrastos (28 viagens) foi encontrado um total de 55 espécies de peixes compreendidos em 21 famílias, sendo em média 10,2 espécies por arrasto (Tab. 3).

Segundo Allsopp (1983), a ictiofauna acompanhante tropical é composta de 70 a 200 espécies. Em estudo realizado no litoral colombiano (Carranza-Fraser \& Grande, 1982) foi encontrada uma média de 18 espécies de peixes, por lance.

Carranza-Fraser \& Grande (op. cit.), apontam a estação do ano como uma das causas responsáveis pela variação da captura da ictiofauna acompanhante na pesca do camarão. Este mesmo fato foi por nós constatato podendo-se notar na Figura 2 uma variação média do índice de diversidade da fauna de peixes em função da época do ano. 0 mesmo padrão de variação de diversidade de ictiofauna, apenas com pequena defazagem no tempo, foi obtido por Paiva Filho (1982), em estudo realizado no estuário de São Vicente, região próxima à baía de Santos.

Embora sem uma tendência mais definida a produção por unidade de esforço (PUE) do total capturado varia em função da estação do ano (Fig. 1).

Analisando-se a quantidade de pescado desembarcado em Santos, (Instituto de Pesca, 1981-1983), verifica-se a mesma tendência de variação do índice de diversidade e da produção por unidade de esforço, ou seja, há nítida diminuição da produção nos meses frios (inverno-primavera). 


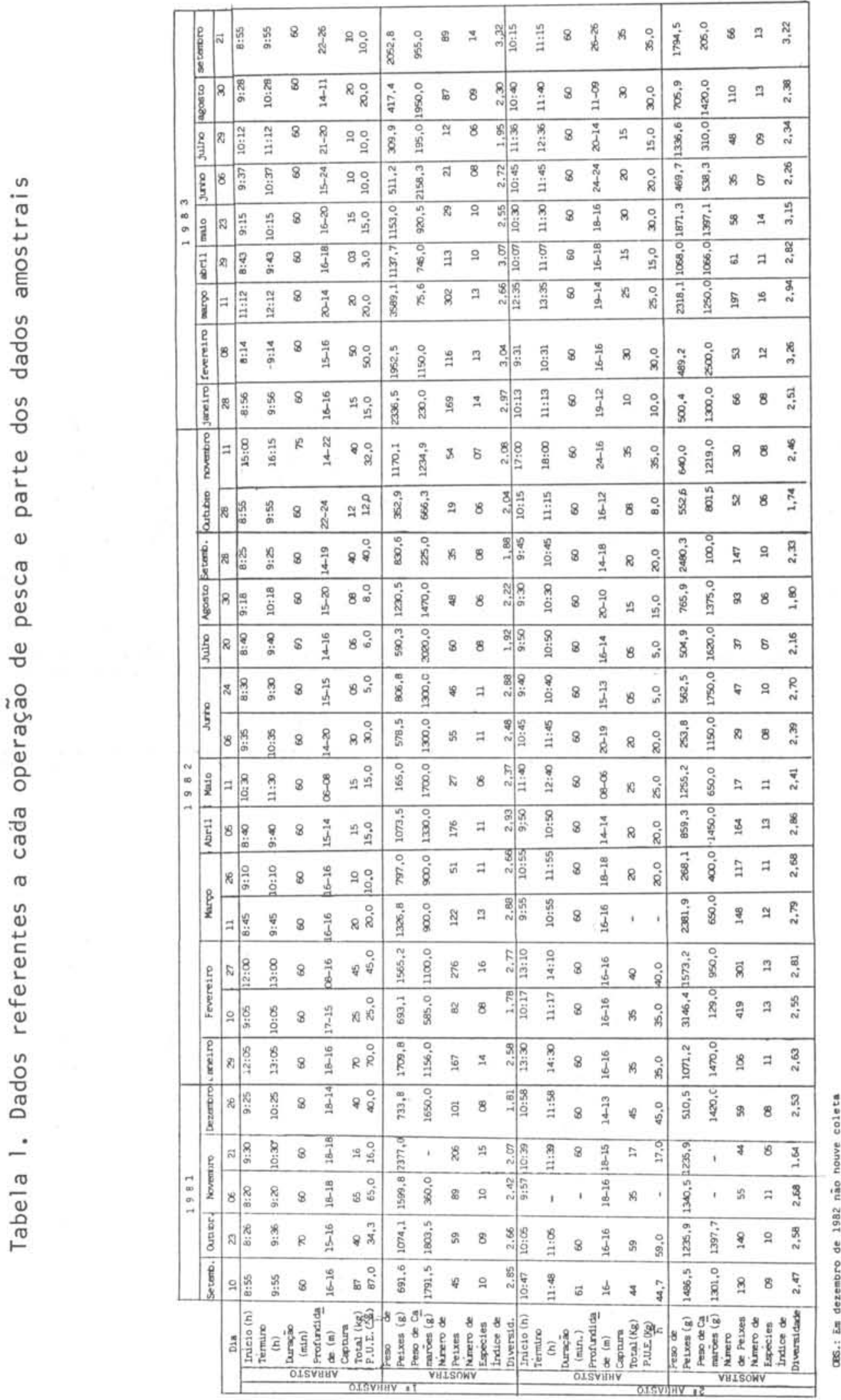




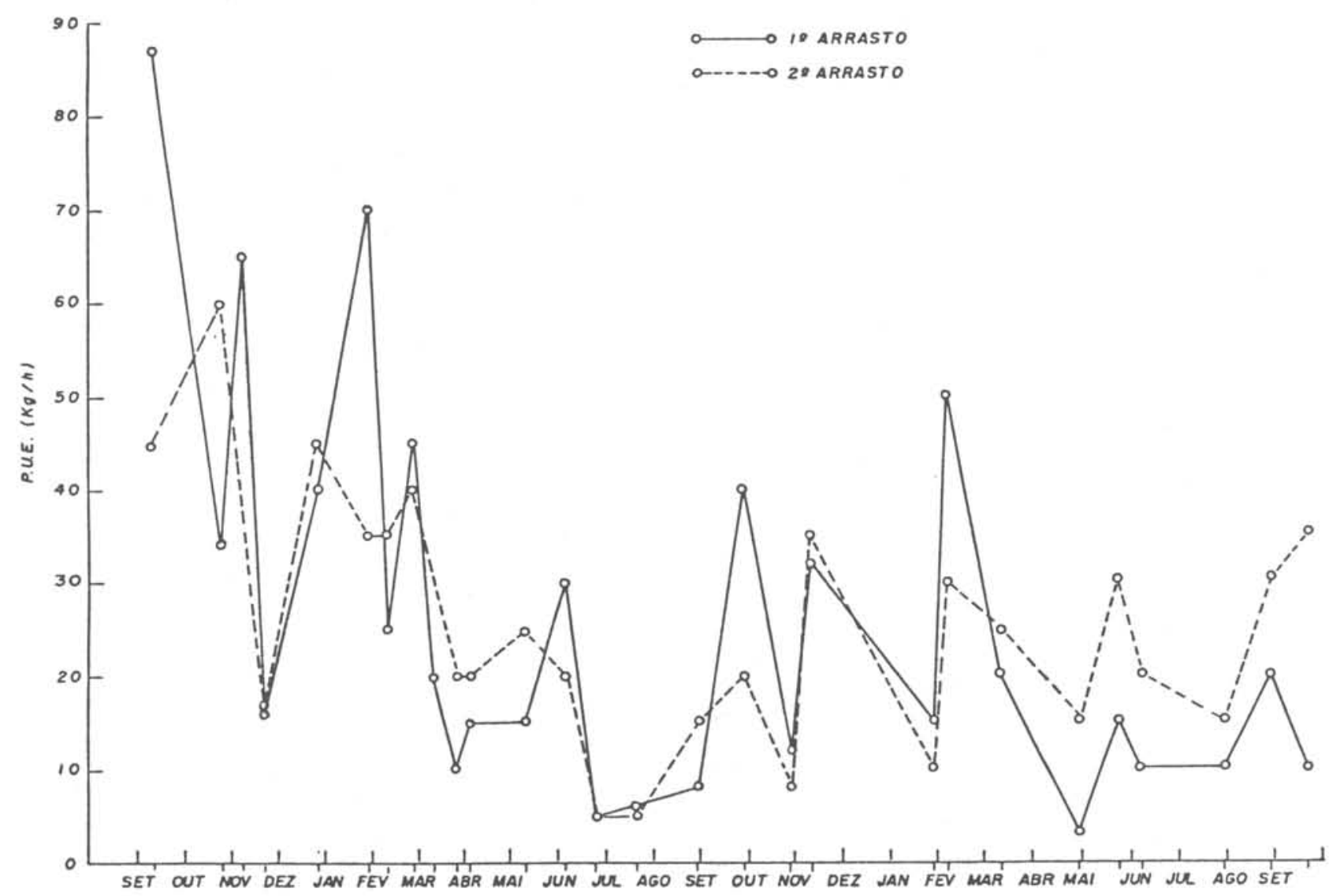

Fig. 1. Produção por unidade de esforço $(\mathrm{Kg} / \mathrm{h})$.

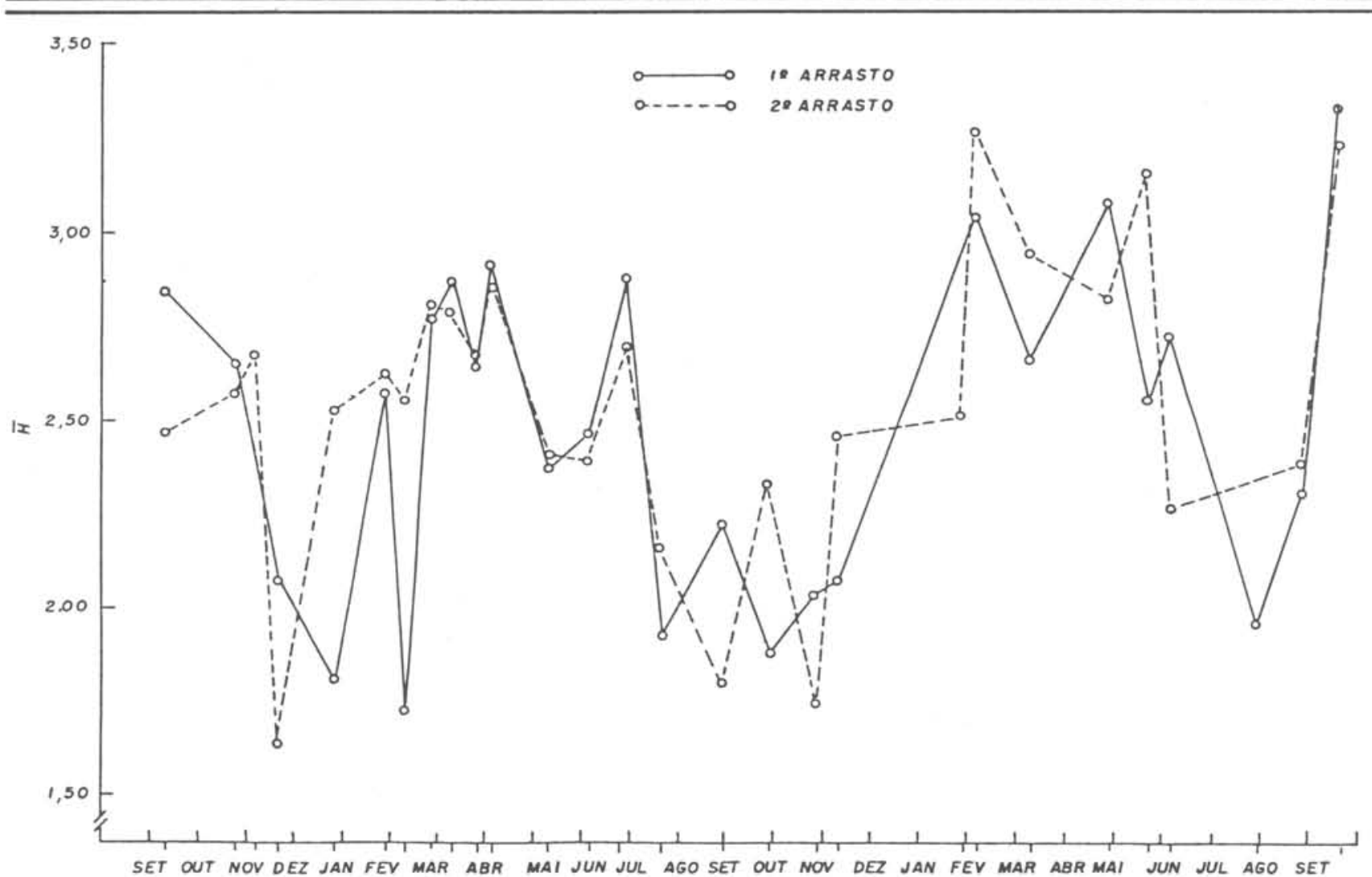

Fig. 2. Tndice de diversidade de Shannon-Wiener. 


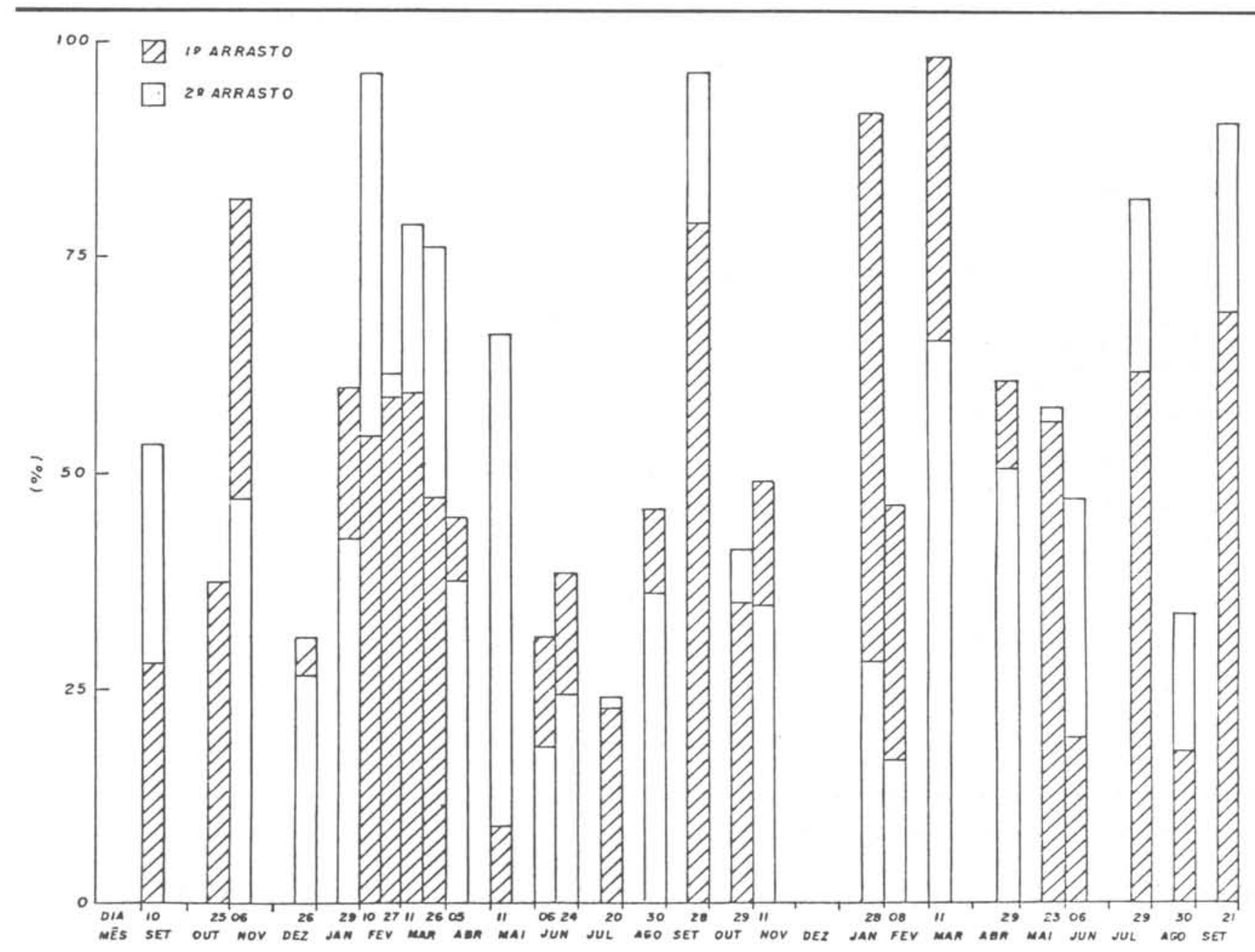

Fig. 3. Porcentagem do peso de peixes em relação ao peso total capturado (camarão e peixe).

A espécie que ocorreu em maior número e peso, $19,7 \%$ e $26,0 \%$ respectivamente, foi Paralonchurus brasiliensis (Maria-Luiza) (Tab. 2).

As seis espécies que ocorreram em maior número e peso somaram $69,7 \%$ e $58,0 \%$, respectivamente do total analisado. Destas, cinco espécies ou $53,3 \%$ em peso são de família Sciaenidae (64,1\% do total de espécies, em peso, são Sciaenidae $(\mathrm{Tab} .2))$. No litoral norte-americano e no Golfo do México, num estudo de Pellegrin Jr (1983) sobre a ictiofauna acompanhante do camarão, a família Sciaenidae foi predominante correspondendo a $53,1 \%$ e $43,4 \%$ em peso, respectivamente.

Segundo Slavin (1983), aproximadamente $50 \%$ da fauna acompanhante do camarão é constituída por 3 a 5 espécies e $75 \%$ de 7 a 10 espécies, de modo geral. No presente estudo, a marca dos $50 \%$ está entre 3 a 4 espécies e aproximadamente $75 \%$ corresponde a 7 espécies em peso.

Dentre as dez espécies mais abundantes, em peso, muitas têm grande importância comercial como a corvina (Micropogonias furnieri) e a pescada-foguete (Macrodon ancylodon) (Tab. 2).

A relação entre o camarão sete-barbas e a ictiofauna acompanhante, em peso, foi de $1: 1,08$, embora ocorressem grandes variações ao longo do ano (Tab. 3).

Existe uma grande variação no volume da pesca acompanhante capturada, na pesca mundial (Allsopp, 1983).

Se tomarmos como significativa a relação camarão/ictiofauna acompanhante obtida $(1: 1,08)$ podemos estimar, sem considerar uma pequena porcentagem de peixes com tamanho comercializável que são vendidos como "mistura", em aproximadamente 5000 toneladas de pei- 
Tabela 2. Espëcies mais abundantes

\begin{tabular}{|c|c|c|c|c|c|}
\hline Espëcie & Familia & $\mathrm{N}$ & $W(g)$ & $2 N$ & $\approx w t$ \\
\hline Paralonchurus brasiliensis & Sciaenidae & 1100 & 17729.5 & 19.7 & 26,7 \\
\hline Isopisthus parvipinnis & Sciaenidae & 895 & 4907,1 & 16,0 & 7,4 \\
\hline stellifer brasiliensis & Sciaenidae & 597 & 4786,2 & 10,7 & 7,2 \\
\hline Stelfifer rastrifer & Sciaenidae & 462 & 5051,4 & 8,3 & 7,6 \\
\hline Pellona harroweri & Clupeidae & 446 & 3107,6 & 8,0 & 4,7 \\
\hline Macrodon ancylodon & Sciaenidae & 392 & 2941,5 & 7,0 & 4,4 \\
\hline Anchoa bilifera & Engraulidae & 219 & 1024,4 & 3,9 & 1,5 \\
\hline Selene setapinnis & Carangidae & 154 & 1289,4 & 2,8 & 1,9 \\
\hline Harengula clupeola & Clupeidae & 152 & 2869,9 & 2,7 & 4,3 \\
\hline Trichiurus lepturus & Trichiuridae & 127 & 2753,2 & 2,3 & 4,1 \\
\hline Chirocentrodon bleekerianus & Clupeidae & 99 & 407,1 & 1,8 & 0,6 \\
\hline Micropogonias furnieni & Sciaenidae & 97 & 2826,4 & 1,7 & 4,2 \\
\hline Porichthys porosissimus & Batrachoididae & 87 & 2896,0 & 1,6 & 4,3 \\
\hline
\end{tabular}

Tabela 3. Nümero mëdio, mínimo e mäximo de espécies por arrasto e proporção média, mínima e mäxima do peso total capturado de camarão/peixe

\begin{tabular}{l|cc}
\hline Relaçöes & $\begin{array}{c}\text { Húmero de } \\
\text { especies/orresto }\end{array}$ & Camaräolpelxe \\
\hline médio & 10,2 & $1: 1.08$ \\
minimo & 5 & $1: 0.10$ \\
maximo & 16 & $1: 47.50$ \\
\hline
\end{tabular}

xes mortos e jogados ao mar, por ano. Isto significa que cerca de 200 toneladas de corvina ou pescada-foguete ou mesmo o peixe-espada (Trichiurus lepturus) (Tab. 2) são capturados nessa pesca, sendo a maioria jovens que ainda não atingiram a primeira maturação sexual.

Não podemos deixar de considerar que, como estas pescas de camarão vêm ocorrendo a dezenas de anos, é bem possível que um certo equilíbrio, sob o ponto de vista ecológico, jä tenha sido estabelecido.

\section{Conclusões}

Os resultados obtidos demonstram que:

1 - A ictiofauna acompanhante é constituída por 55 espécies;

2 - Ocorre variação na diversidade e densidade (PUE) da ictiofauna durante o ano;

3 - Em média, a relação, em peso, camarão/ictiofauna acompanhante é de 1:1,08;

4 - Anualmente são capturadas aproximadamente 5000 toneladas de peixes na pesca do camarão sete-barbas sendo que a captura de espécies que, quando maiores, apresentam valor comercial como a corvina, pescada-foguete e o peixe-espada, está ao redor de 200 ton/ano cada.

\section{Referências bibliográficas}

ALLSOPP, W. H. L. 1983. Utilización de la pesca acompañante del arrastre camaroneira: desarrollo futuro. Pesca acompañante del camarón - un regalo del mar: informe de una consulta técnica sobre la utilización de la pesca acompañante del camarón celebrada en Georgetown, Guyana, 27-30 octubre 1981. Otawa, Ont., CIID, p. 32-40. 
CARRANZA-FRASER, J. \& GRANDE, J. M, 1982. Experiencia de México en el aprovechamiento de 1a fauna de acompañamiento del camarón. Proc. Gulf Caribb. Fish. Inst. USA n. 34, p.109-111.

GOOD, I. J. 1950. Probability and the weighing of evidence. Graffin London, in Pielou, E. C. 1966. The measurement of diversity in different types of biological collections. J. Theoret. Biol., 13:131-144.

INSTITUTO DE PESCA. Divisão de Pesca Marítima. 1981-1983. Dados dos quadros estatísticos sobre a produção de pesca marítima no Estado de São Paulo.

IWAI, M. 1971. Pesca exploratória e estudo biológico sobre o camarão na costa Centro-Sul do Brasil do N/Oc. "Prof. W. Besnard" - SUDELPA.

NEIVA, G. de S. 1963. The biology and fishery of the sea-bob shrimp of Santos Bay, Brazil. Proc. Gulf Caribb. Fish. Inst., 16th An. Ses., p. 131-139.

\section{Observações so-} bre a pesca de camarões do litoral Centro-Sul do Brasil - SUDEPE Pesca e Pesquisa, RJ, $2(2): 1-16$.

PAIVA FILHO, A. M. 1982. Estudos sobre a ictiofauna do Canal dos Barreiros, Estuário de São Vicente, SP. Tese de Livre-docência. Universidade de São Paulo, Instituto Oceanográfico, 189p.

PELLEGRIN Jr, G. 1983. Descarte de pescado en la pesquería de camarón en el sudeste de Estados Unidos. Pesca acompañante del camarón - un regalo del mar: informe de una consulta técnica sobre la utilización de la pesca acompañante del camarón celebrada em Georgetown, Guyana, 27-30 octubre 1981. Otawa, Ont., CIID, p.56-60.

PETERKIN, F. A. 1982. Developing a shrimp bycatch utilization program. Proc. Gulf Caribb. Fish. Inst. USA n. 34, p. 112-119.
SANTOS, E. P. dos; NEIVA, G. de S. \& SCHAEFFER, Y. 1969. Dinâmica da população do camarão sete-barbas, Xyphopenaeus kroyeri, (Heller) na baía de Santos SUDEPE, Pesca e Pesquisa, RJ, $2(2): 41-55$.

\& VALENTINI, H. 1971a. Curva de reprodução da população de camarão sete-barbas, Xyphopenaeus kroyeri (Heller) da Baía de Santos. Bolm Inst. Pesca, S Paulo, $1(3): 15-22$.

1971b. Curva de mortalidade da população de camarão sete-barbas, Xyphopenaeus kroyeri (Heller) da Baía de Santos. Bolm Inst. Pesca, S Paulo, 1(5):39-46.

SLAVIN, J. W. 1983. Utilización de la pesca acompañante del camarón. Pesca acompañante del camarón - un regalo del mar: informe de una consulta técnica sobre utilizaciōn de la pesca acompañante del camarōn celebrada em Georgetown, Guyana, 27-30 octubre 1981. Otawa, Ont., CIID. p. 23-31.

STERNIN, V. \& ALLSOPP, W. H. L. 1983. Estrategias para evitar la pesca acompañante en el arrastre camaronero. Pesca acompañante del camarōn-un regalo del mar: informe de una consulta técnica sobre utilización de la pesca acompañante de1 camarón celebrada em Georgetown, Guyana, 27-30 octubre 1981. Otawa, Ont. CIID. p.67-71.

VIEIRA, B. B. 1947. Observações sobre a maturação de Xyphopenaeus kroyeri no litoral de São Paulo. Bolm Mus. Nac. Rio de Janeiro, n.s., Zool., $74,22 \mathrm{p}$. 